\title{
TRÊS FASES RUMO AO DESENVOLVIMENTO SUSTENTÁVEL do reducionismo à valorização da cultura
}

KaRIN VeCChiatti

\begin{abstract}
Resumo: Este artigo apresenta um panorama dos pensamentos que moldaram a forma de se abordar os problemas referentes à sustentabilidade a partir da década de 70. De uma visão estritamente pontual e tecnicista, passou-se por uma ênfase na gestão, atingindo-se, ainda que de forma muito incipiente, uma melhor percepção do papel da cultura.

Palavras-chave:desenvolvimento sustentável; políticas públicas; cultura.
\end{abstract}

\begin{abstract}
This article presents the main thoughts that patterned the sustainability problems' approach in the 70's: a process that started in a strict and technical point of view, passed by a management-like step and reached a good, though incipient, perception of culture role.

Key words:sustainable development; public politics; culture.
\end{abstract}

$\mathrm{N}$ ão é preciso uma investigação detalhada para se dar conta do paradoxo no qual o século XX terminou e o século XXI se inicia: por um lado, o crescimento econômico e a transformação tecnológica sem precedentes, por outro, a dramática condição social de inúmeras pessoas, além de problemas ambientais assustadores. Se ao longo desses anos desenvolveu-se um aparato científico-tecnológico capaz de resolver grande parte dos principais problemas ecológicos, ficou também cada vez mais notável a incapacidade das formas sociais organizadas de se apropriarem desses meios.

Uma das conclusões óbvias que pode ser extraída desse quadro de contrastes é que o crescimento econômico, por si só, não traz automaticamente o desenvolvimento. Na prática, a equação que relaciona crescimento e desenvolvimento ainda não está com suas variáveis equilibradas; ela ainda desafia os economistas questionando se o desenvolvimento socialmente justo e ambientalmente sustentável estaria realmente na contramão do crescimento econômico.
Para um dos grandes nomes no debate, o economista Ignacy Sachs, o crescimento econômico, se colocado a serviço de objetivos socialmente desejáveis e repensado de forma adequada, de modo a minimizar os impactos ambientais negativos, continua sendo uma condição necessária para o desenvolvimento. Aliás, taxas significativas de crescimento são necessárias, uma vez que é muito difícil redistribuir bens e renda numa economia estagnada (SACHS, 2001). A importância, então, estaria em se distinguir diferentes tipos de crescimento. A variedade mais freqüente é a "selvagem", com custos sociais e impactos ambientais insuportavelmente altos. Hoje, sabe-se que esse tipo de crescimento impulsionado pelo mercado é inaceitável dos pontos de vista social e ambiental, além de não estabelecer, por si só, uma situação empregatícia satisfatória. Os mercados são por demais míopes para transcender os curtos prazos e cegos para quaisquer considerações que não sejam lucros e eficiência "smithiana" de alocação de recursos (SACHS, 2002). 
As variedades "benignas" de crescimento, em contrapartida, requerem justamente o pensamento contrário. Ou seja, a consideração da sustentabilidade do desenvolvimento requer que nosso horizonte de tempo seja expandido (MORIN, 2001). Enquanto os economistas estão habituados a raciocinar em termos de anos, no máximo décadas, a escala de tempo da ecologia abrange séculos. Além disso, a expressão "desenvolvimento sustentável" não adverte somente dos danos causados por poluição e erosão do ambiente físico de um lado e da pobreza do outro, como se fossem dois fenômenos independentes. O tipo "benigno" de desenvolvimento desejável propõe uma conciliação entre o desenvolvimento e o crescimento econômico, sendo, simultaneamente, sensível à dimensão social, ambientalmente prudente e economicamente viável.

É claro que essa tarefa de conciliação não é fácil. Mas uma observação mais atenta ressalta que a busca pela sustentabilidade requer a articulação entre três "registros ecológicos": do meio ambiente, das relações sociais e da subjetividade humana (GUATTARI, 1990). Em outras palavras, essa proposta sugere que o rumo à sustentabilidade é:

- incompatível com o jogo sem restrições das forças de mercado;

- dependente de um aparato tecnológico eficiente;

- dependente, em grande parte, das ações geradas a partir de percepções individuais e culturais da sociedade.

É justamente nesse ponto em que reside a importância das políticas públicas. Somente uma articulação éticopolítica entre essas dimensões poderia direcionar uma revolução social e cultural, reorientando a produção de bens materiais e imateriais, reconciliando o crescimento econômico com as formas de desenvolvimento sustentável.

Essa realidade ainda está distante. Mas não se pode negar que avanços foram obtidos no campo das políticas públicas desde os anos 70. O início da tomada de consciência social sobre os inúmeros problemas ambientais naquela década conduziu à redação de relatórios, cujos objetivos eram essencialmente esboçar avaliações globais, fazer emergir as questões consideradas urgentes e formular recomendações imediatas às diferentes instâncias do poder político. Desde então, o conceito de meio ambiente como objeto de pesquisa e de políticas públicas aos poucos está se alterando, de forma que seja possível identificar ao longo desse período três fases no pensamento. Esses períodos podem ser caracterizados por diferentes maneiras de abordar os problemas referentes à sustenta- bilidade, que por sua vez se refletem na formulação e implantação de políticas públicas. São eles:

- período inicial, de transição de uma visão pontual para uma visão abrangente dos problemas ambientais;

- período de ênfase na gestão, na informação e na articulação territorial;

- período incipiente com a atenção voltada para a cultura.

Essa divisão não indica, necessariamente, que as idéias e as políticas públicas características da primeira fase não vigorem nos anos seguintes, como é o caso, por exemplo, do Código Florestal, tampouco que uma preocupação atual não estivesse presente anos atrás. Contudo, a compreensão dessas três fases (aqui brevemente apresentadas), segundo a abordagem dominante em cada uma delas, parece ser fundamental para traçar um panorama do caminho percorrido e identificar o quão longe se está ainda de uma conciliação entre o crescimento econômico e o desenvolvimento sustentável.

\section{DO PONTUAL AO ABRANGENTE}

A primeira fase reflete a noção de que os problemas referentes à sustentabilidade não podem ser analisados de forma pontual. O embrião dessa idéia começou a ser formado ainda na década de 70, tendo como marco a Conferência de Estocolmo, primeira reunião oficial a tratar das questões ambientais no âmbito mundial. Organizada pela ONU, a conferência reuniu 113 países e 250 organizações não-governamentais. Tinha como objetivos: fazer um balanço dos problemas ambientais em todo o mundo; buscar soluções e novas políticas governamentais no sentido de reduzir o grande número de problemas causados pelo desenvolvimento das sociedades, tais como poluição, deterioração dos ambientes e limitação dos recursos naturais; discutir a urbanização acelerada, mal concebida e caótica; debater o caráter global dessas perturbações de origem humana.

Nessa época, entretanto, acreditava-se que a modernização dos processos produtivos seria suficiente para resolver os problemas ambientais e que a solução dependia apenas da legislação e de técnicas de controle de poluição (estratégia que ficou conhecida como "comando-controle"), conforme postulavam os representantes dos países industrializados. Mas, ao longo do tempo, percebeu-se que essa é uma visão tecnicista e reducionista, sendo necessária uma abordagem mais ampla dos problemas e das soluções, discutindo-se, necessariamente, o modelo de desenvolvimento internacional. 
O conceito de desenvolvimento sustentável surgiu somente na década de 80 e foi consagrado em 1987 pela Comissão Mundial sobre o Meio Ambiente - CMMA das Nações Unidas, conhecida como Comissão Brundtland, que produziu um relatório considerado básico:

[...] um processo de transformação no qual a exploração dos recursos, a direção dos investimentos, a orientação do desenvolvimento tecnológico e a mudança institucional se harmonizam e reforçam o potencial presente e futuro [...] é aquele que atende às necessidades do presente sem comprometer a possibilidade de as gerações futuras atenderem às suas próprias necessidades (IBGE, 2002).

Esse conceito foi decisivo para se repensar as dimensões do desenvolvimento e para a conscientização da sociedade, questionando se as práticas econômicas e sociais desenvolvidas até então não estavam limitando a capacidade dos ambientes naturais de suportar a vida no planeta. Assim, começava a se discutir se o modelo de desenvolvimento adotado seria insustentável ao logo do tempo, comprometendo a vida de futuras gerações (WEBER, 1997).

Foi com esse pano de fundo que a revista São Paulo em Perspectiva (FUNDAÇÃO SEADE, 1989) publicou seu primeiro volume referente aos problemas ambientais. Os artigos chamam a atenção para a ocupação não-planejada e predatória do Estado de São Paulo, para o conflito gerado a partir do uso da água nas diferentes bacias hidrográficas do Estado e para o uso indiscriminado de agrotóxicos no combate de pragas agrícolas. Os textos alertam também para o processo de transformações da metrópole, contribuindo para a deterioração da qualidade de vida da população, sobretudo de sua saúde. Ficava cada vez mais óbvia a gravidade dos altos níveis de poluição hídrica e atmosférica em diversos municípios da Região Metropolitana de São Paulo, além da formação das ilhas de calor e das enchentes, em grande parte provocadas pela escassez de áreas verdes.

Por trás do alerta, entretanto, os artigos trazem a idéia de que, à medida que os problemas ambientais se agravavam, havia necessidade de se desenvolver metodologias e teorias que enxergassem o objeto de pesquisa de forma mais ampla. Os textos relatam que os problemas anteriormente analisados e tratados de forma pontual tanto do ponto de vista científico, como do ponto de vista da administração pública (poluição hídrica e atmosférica, desmatamento, etc.), começavam a ser vistos a partir de uma óptica distinta. Sobretudo porque a solução para gran- de parte dos problemas ambientais não estava somente na eficiente implantação de algumas leis restritivas. Apesar da importância da tecnologia, das restrições ambientais e das multas aplicadas às ações que tinham impacto negativo sobre o meio ambiente, um outro componente não poderia ser descartado: a gestão a longo prazo, que em grande parte depende dos variados tipos de relações microssociais. Assim, aplicar o mesmo tipo de multa a um agricultor do Estado de São Paulo ou a uma madeireira no Estado do Pará, sem um suporte informacional e, posteriormente, um incentivo a atividades sustentáveis, dificilmente resolveria o problema a longo prazo. Na tentativa de solucionar esses impasses (muitos presentes até hoje), o foco voltouse para a gestão, sendo a qualidade do meio ambiente vista a partir de um enfoque territorial.

\section{GESTÃO E ARTICULAÇÃO TERRITORIAL}

A elaboração da Agenda 21, no início da década de 90 , contribuiu para impulsionar a criação de abordagens territoriais a partir de redes de comunicação, buscandose soluções para os problemas referentes à sustentabilidade e à tentativa de conciliar o crescimento econômico com o desenvolvimento. Os princípios do desenvolvimento sustentável formaram a base da Agenda 21, um documento aprovado por mais de 180 países durante a conferência das Nações Unidas sobre meio ambiente no Rio de Janeiro, em 1992. Desde então, esses preceitos têm sido progressivamente incorporados à agenda de numerosos países, principalmente no âmbito local. Assim, ampliou-se a consciência de que os problemas ambientais não são assunto de um setor restrito da economia ou da sociedade nem se restringem aos danos causados ao ambiente físico natural, mas envolvem as relações sociais em um determinado território.

Como reflexo da Agenda 21, surgiu uma grande necessidade de geração e divulgação de informações para a resolução de problemas locais. Um dos melhores exemplos de ações resultantes dessa percepção são os programas estaduais de gerenciamento de microbacias hidrográficas, introduzidos tanto no Estado de São Paulo como em outros Estados brasileiros. De acordo com essas iniciativas, a responsabilidade de gerenciamento dos recursos hídricos de uma região está a cargo das agências estaduais de meio ambiente e recursos hídricos e também dos municípios, representados por governos locais e agentes da sociedade civil. Embora o incentivo às iniciativas locais por si só não seja suficiente, sem sólidas estruturas locais 
participativas e democratizadas não há financiamentos externos ou de instituições centrais que produzam resultados efetivos.

A idéia do desenvolvimento local transferiu o foco de atenção da poluição para a gestão, da ênfase na molécula poluidora para a educação ambiental e da técnica de comando-controle para as políticas territoriais. A transição para uma abordagem territorial e informacional da sustentabilidade é extremamente importante para a eficiência na geração e implantação de políticas públicas, pois o potencial de desenvolvimento de um país depende, principalmente, de sua capacidade cultural de pensar de forma endógena sobre seus futuros desejáveis (SACHS, 2001). Essa característica, por sua vez, está ligada ao grau de percepção, ao conhecimento e à consciência que a sociedade tem de sua história, sua situação presente e seu rumo futuro. Tal estado de consciência coletiva requer que os indivíduos enxerguem suas responsabilidades por fazerem parte de um conjunto maior de ações, como acontece com as propostas de desenvolvimento local. Idealmente, essa capacidade impulsiona a geração de políticas públicas nas mais diferentes esferas do desenvolvimento. $\mathrm{Na}$ maioria das vezes, no entanto, uma combinação de fatores históricos e culturais faz com que os indivíduos privilegiem sua sobrevivência ou suas necessidades individuais, em vez de tomarem parte em um todo, o que diminui a eficiência da criação e implantação das políticas públicas.

Outro mérito referente às políticas territoriais reside na possibilidade de promoção da convivência com a natureza. A conservação da biodiversidade não pode ser equacionada com a opção do não-uso dos recursos naturais. Por importante que seja, a instituição das reservas naturais praticamente intocadas é apenas uma das estratégias de conservação. O conceito de reservas de biodiversidade da Unesco nasceu da compreensão de que a conservação da biodiversidade deve estar em harmonia com as necessidades das sociedades que vivem em determinado ecossistema (SACHS, 2002). Daí a necessidade de se enxergar essas regiões a partir do ponto de vista da gestão do território, que inclui os recursos naturais, as atividades econômicas, o acesso às condições básicas de saúde e educação e o respeito a costumes e tradições.

Ainda vive-se uma fase que requer maior aplicação das políticas territoriais, pois em diversas áreas esse tipo de enfoque ainda não desempenhou seu papel por completo. Muitas regiões rurais paulistas, por exemplo, poderiam beneficiar-se enormemente desse tipo de gestão. É importante lembrar que o meio rural não se restringe à agricultura, mas constitui efetivamente um território permeado por relações sociais, não se reduz a um setor da economia. A valorização do meio rural pode ser um dos caminhos importantes para atenuar os explosivos problemas que atingem hoje as cidades, sobretudo nos países e nas regiões onde o peso da população que vive no campo é importante. A valorização do campo não implica a rejeição do papel dinamizador que as cidades desempenham na sociedade (ABRAMOVAY, 2000; VEIGA, 2002). Significa incentivar, por meio de políticas territoriais, um grupo de municípios rurais a se articular e definir seu rumo, vivendo, conservando e recuperando suas paisagens menos afetadas pelas mazelas do desenvolvimento. Isso é muito diferente de pregar uma forma de "volta ao meio rural" ou de sugerir a fixação do homem no campo. A chave está na ampliação dos horizontes da vida social, econômica e cultural dos habitantes desse meio, o que leva à terceira fase rumo ao desenvolvimento sustentável: a cultura.

\section{A CULTURA COMO FATOR DE SUSTENTABILIDADE}

A ênfase na cultura como fator de sustentabilidade ainda é muito recente especialmente nas políticas públicas. $\mathrm{O}$ seu desenvolvimento pode ser um importante fator no período em que vivemos, pois não se restringe a um segmento específico, mas permeia diversas ações da sociedade; lida com a criatividade que transita entre o novo e o antigo e impulsiona a sociedade a construir um quadro de referência com relação a seu futuro. Apesar de raramente pensadas em termos de sustentabilidade, as políticas culturais são de suma importância, porque suas ferramentas de intervenção geralmente se aproximam da subjetividade humana, o terceiro registro ecológico sugerido por Guattari (1990) e componente fundamental da articulação ético-política capaz de conciliar o desenvolvimento ao crescimento econômico.

Sabe-se que a cultura muda muito lentamente. Apesar de a perspectiva do desenvolvimento sustentável pressupor uma atitude psicológica essencialmente futurista, é praticamente impossível que qualquer geração seja capaz de prever todas as contingências implicadas na evolução cultural. Mas, à medida que o indivíduo se conscientiza da repercussão de seus atos nas gerações futuras e se preocupa com isso, desenvolve-se uma preocupação através das gerações capaz de enxergar uma responsabilidade 
comum. Para alcançar esse estado ideal, entretanto, o desenvolvimento cultural deve partir do reconhecimento dos cenários nos quais os atores sociais interagem, constroem espaços, mudam os valores e os "olhares" sobre a vida em sociedade. A partir daí, incentivar o desenvolvimento da cultura não corresponde a apenas realizar produtos com viabilidade de mercado que dêem visibilidade a empresas, muito menos a vender a cultura como um produto a ser consumido. Trata-se de compreender a cultura como um processo de criação de significados que oferecem sentido ao modo de vida das comunidades humanas.

Pensar na cultura como fator de desenvolvimento significa valorizar identidades individuais e coletivas, promover a coesão em comunidades e levar em consideração que as características da cultura podem ser um fator de crescimento em determinado território, como é o caso de diferentes regiões rurais com relação aos seus produtos agrícolas, seus costumes e paisagens aproveitadas pelo turismo. Assim, não há fronteiras territoriais. A cultura é tão essencial em grandes metrópoles como em áreas rurais. Em cada local, diferentes agentes são envolvidos, com tarefas e formatos variados e, conseqüentemente, resultados distintos. Mas os processos são sempre muito similares, envolvendo, por meio de parcerias de médio e longo prazos, os agentes públicos, privados e do terceiro setor.

O papel fundamental da cultura pode beneficiar o desenvolvimento de outras políticas públicas, acentuando sua eficiência, tanto nos aspectos econômicos, como nas dimensões social e ambiental (RUIZ, 2003). Diversos trabalhos desenvolvidos pela Unesco (1995) ressaltam que, especialmente nos chamados "territórios periféricos" da Europa Oriental, o desenvolvimento não caminha sem uma especial ênfase ao desenvolvimento da cultura local, ou seja, é preciso reforçar a consciência dessas culturas em relação ao seu desenvolvimento.

Hoje, as politicas culturais devem contribuir para gerar pertencimentos a partir do resgate da auto-estima individual e coletiva. Sem a auto-estima não é possivel o desenvolvimento humano [...] e sem o pertencimento, não há o desenvolvimento integral,

ressalta o Instituto Pólis (FARIA, 1999) no relatório produzido a partir de um seminário em São Paulo, que apresentou como diversas ações culturais e artísticas são capazes de beneficiar o desenvolvimento humano, comunitário e municipal. A área produtiva, as redes de infraestrutura e de serviços não funcionam de maneira adequada se não houver investimento no ser humano, em sua formação, saúde, cultura, lazer e informação.

Incentivar o desenvolvimento da cultura em um país como o Brasil ainda é visto como um elemento supérfluo, de "perfumaria", e pode ser considerado um trabalho dificílimo e infinito, devido a verbas restritas, incapazes de atender à efervescência de incontáveis manifestações. Mas a proposta fica mais clara se pensarmos que o desenvolvimento e sua conciliação com o crescimento econômico não se darão a partir da implantação de um elemento específico da cultura, mas sim pela interação entre diferentes centros de influência (as artes, as escolas, as instituições públicas e privadas, por exemplo) e pelas políticas públicas, como balizadoras e direcionadoras das ações governamentais, certamente capazes de impulsionar o aperfeiçoamento e a interação desses centros.

$\mathrm{O}$ incentivo à formação de público para a Orquestra Sinfônica do Estado de São Paulo, a Pinacoteca e o Museu de Arte Sacra na capital paulista, a recuperação da Estação da Luz e a implantação de um museu destinado à língua portuguesa, além das diversas ações da Secretaria de Estado da Cultura procurando transformar São Paulo em um Estado de leitores são exemplos de interação entre esses centros de influência. Eles demonstram que as políticas culturais podem e devem desempenhar um papel significativo na promoção da sustentabilidade, o qual pode ser entendido como a recriação de uma teia de comunicação no espaço público.

Existem diferentes frentes nas quais as políticas públicas culturais podem agir, segundo essa óptica. Os trabalhos artísticos compõem uma delas. A arte, entretanto, é exemplar quando confere um novo tipo de signo à existência e

opera transformações a partir de linguagens que valorizam a vida como um todo e criam um outro discurso, diferente daquele que já não sabe comunicar-se ou comunica-se mal com a sociedade (FARIA, 1999, p. 15).

A arte é constituída da vida social e impulsiona relações entre pessoas e grupos, renovando vivências, laços de solidariedade, criando imagens e poéticas imprescindíveis para o conhecimento de si mesmo e do outro e contribuindo para a criação de um rico imaginário local apoiado nas raízes e na criatividade coletivas.

Ainda pouco explorados nas ações promotoras da sustentabilidade, os trabalhos artísticos que tocam o imaginário e a subjetividade humana fazem parte de um importante registro ecológico de nossas ações. Isso porque as 
novas idéias são visões imaginativas originais, não somente porque elas envolvem imagens particulares inéditas, mas no sentido de que envolvem mudanças na nossa visão de mundo, a forma geral pela qual percebemos a vida. A imagem da máquina, por exemplo, transformou a visão de mundo profundamente durante a Renascença, sobretudo porque máquinas estão sob o controle humano. E a idéia de se poder separar essa máquina (o mundo) em partes é o que fez a ciência parecer possível. E precisava parecer possível na imaginação antes que qualquer pessoa começasse efetivamente a praticá-la (MIDGLEY, 2001). O mesmo pode ser válido para o desenvolvimento sustentável: primeiro é preciso imaginá-lo possível.

\section{PRÓXIMOS PASSOS}

Se uma conciliação entre o crescimento econômico e o desenvolvimento sustentável ainda parece remota, enxergar e compreender o caminho percorrido pode ser importante, principalmente para a formulação de políticas públicas que tenham como propósito incentivar formas sustentáveis de desenvolvimento. Esse percurso indica que é necessária uma visão abrangente da sustentabilidade e que as políticas públicas sejam integradas dentro de uma perspectiva de longo prazo de gestão territorial, na qual a cultura seja um importante elemento impulsor. Em outras palavras, além de impor leis restritivas, as políticas públicas devem procurar criar um ambiente favorável à sustentabilidade ao direcionarem as ações do Estado referentes a seus mais diversos setores de atuação.

Como o século XXI pede urgência na derrubada de contradições que marcaram o final do século passado, não se pode tardar a direcionar o foco das políticas públicas para a sustentabilidade e para a geração de informações, ambas partes de um conjunto de objetivos que pautam e condicionam a ação governamental. À medida que as políticas públicas contemplarem propostas que articulem o meio ambiente, a sociedade e a subjetividade humana, o abismo que separa o crescimento econômico do desenvolvimento poderá não parecer tão grande e a sustentabilidade, não tão distante.

\section{REFERÊNCIAS BIBLIOGRÁFICAS}

ABRAMOVAY, R. Funções e medidas da ruralidade no desenvolvimento contemporâneo. Rio de Janeiro: Ipea, jan. 2000. (Texto para discussão, n.702).

FARIA, H. (Org.). Seminário Desenvolver-se com Arte. São Paulo: Instituto Pólis, 1999.

FUNDAÇÃO SEADE. São Paulo em Perspectiva, São Paulo, v. 3, n. 4, out./dez. 1989.

GUATTARI, F. As três ecologias. Campinas: Papirus, 1990.

IBGE. Indicadores de desenvolvimento sustentável. Rio de Janeiro: 2002.

MIDGLEY, M. Science and Poetry. London: Routledge, 2001.

MORIN, E. O método. São Paulo: Editora Sulina, 2001. 5 v.

RUIZ, J. P. Cultural Development, Public Policies and Local Strategies. In: Local Cultural Strategies Development in South-East Europe, Building on Practice and Experience. Background paper. Policies for Culture Regional Workshop. Bucareste, 8-10 Maio, 2003.

SACHS, I. Repensando o crescimento econômico e o progresso social: o papel da política. In: ABRAMOVAY, R. et al. (Orgs.). Razões e ficções do desenvolvimento. São Paulo: Editora Unesp/Edusp, 2001 .

Caminhos para o desenvolvimento sustentável. Rio de Janeiro: Garamond, 2002.

UNESCO. World Commission on Culture and Development. Relatório Our Creative Diversity. Paris: Unesco Publishing, 1995.

VEIGA, J. E. Cidades imaginárias: o Brasil é menos urbano do que se calcula. Campinas: Autores Associados, 2002.

WEBER, J. Gestão dos recursos renováveis: fundamentos teóricos de um programa de pesquisa. In: VIEIRA, P.F.; WEBER, J. (Org.). Gestão dos recursos naturais renováveis e desenvolvimento: novos desafios para a pesquisa ambiental. São Paulo: Editora Cortez, 1997.

Karin VeCChiatti: Engenheira Agrônoma, Mestre em Ciência Ambiental pela Procam/USP. 